Supporting Information for

\title{
Micromechanical Landscape of Three-Dimensional Disordered Graphene Networks
}

YinBo Zhu, YongChao Wang, Bao Wu, ZeZhou He, Jun Xia, HengAn Wu* CAS Key Laboratory of Mechanical Behavior and Design of Materials, Department of Modern Mechanics, CAS Center for Excellence in Complex System Mechanics, University of Science and Technology of China, Hefei 230027, China

*Corresponding author. wuha@ustc.edu.cn (HengAn Wu) 


\section{Computational methods and models.}

Models of DGNs. DGNs can be obtained in annealing-MD simulations. Refs. 1-5 and our recent work reported the detailed methods of modeling DGNs. ${ }^{1-6}$ The file of EDIP force field was kindly provided by Prof. Marks. ${ }^{5}$ Note that, compared to the simulations in Refs. 1-3, we modified the simulation methods to obtain better and reasonable DGN structures. ${ }^{6}$ Since the EDIP force field does not include long-range interaction between carbon atoms,${ }^{5}$ we then used the AIREBO potential within the NVT ensemble to avoid the inappropriate interlayer spacing in some local microstructures. ${ }^{6}$ Scheme S1 illustrates several models of DGNs with different densities. In this study, we considered the density ranging from 0.1 to $1.64 \mathrm{~g} / \mathrm{cm}^{3}$, and each model contains $\sim 10^{5}$ carbon atoms. The size of model is changed with the density due to that lower-density DGNs exhibit more connected pores. Size parameters for different models are listed in Table S1. In each model, the amount of $s p^{2}$ carbon atoms is $\sim 97 \%$, which is very close to the experimental measurements of PyC. Few $s p^{3}$-bonded atoms can be found in DGNs due to the necessity of out-of-plane topological defects in 3D continuous networks, ${ }^{6}$ such as Y/T junctions, screw dislocations, and distorted edges. ${ }^{1}$ The amount of $s p^{3}$ atoms increases with the increase of DGN density due to the distorted network.

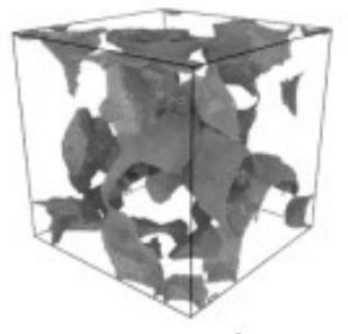

$0.10 \mathrm{~g} / \mathrm{cm}^{3}$

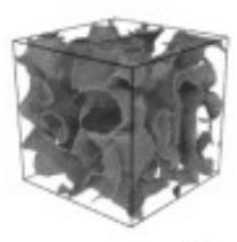

$0.33 \mathrm{~g} / \mathrm{cm}^{3}$

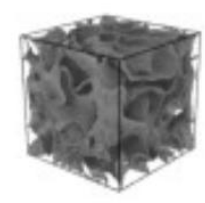

$0.56 \mathrm{~g} / \mathrm{cm}^{3}$

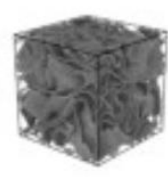

$1.12 \mathrm{~g} / \mathrm{cm}^{3} \quad 1.54 \mathrm{~g} / \mathrm{cm}^{3}$

Scheme S1. Several main models of DGNs in our simulations.

Table S1. Size parameters for DGNs with various densities.

\begin{tabular}{cc}
\hline Density $\left(\mathrm{g} / \mathrm{cm}^{3}\right)($ ref. 6) & Side length $(\AA)$ \\
\hline 0.10 & 270 \\
0.22 & 210 \\
0.33 & 185 \\
0.45 & 168 \\
0.56 & 156 \\
0.68 & 146 \\
0.79 & 139 \\
0.90 & 134 \\
1.01 & 128 \\
1.12 & 124 \\
1.24 & 120 \\
1.35 & 117 \\
1.46 & 114
\end{tabular}


MD simulations of mechanics. All MD simulations were carried out using the LAMMPS program. ${ }^{7}$ Note that, the EDIP force field cannot be used in mechanical simulations due to the non-physical strengthening found in the tensile test of graphene monolayer. Therefore, in subsequent simulations, the inter-atomic force among carbon atoms was simulated by the adaptive inter-molecular reactive empirical bond order (AIREBO) potential, ${ }^{8}$ and the cut-off parameter was adjusted to $2.0 \AA .{ }^{9}$ The modified AIREBO potential can well predict the in-plane modulus, tensile strength and fracture strain of graphene and other carbon materials, ${ }^{10}$ which was usually used to simulate the mechanical response of $s p^{2}$-dominated carbon materials and structures. ${ }^{11-13}$ During all simulations, periodic boundary conditions were used in three dimensions of the simulation box. The time step used for integration was $1 \mathrm{fs}$. Before the deformation loading, the models were relaxed in an NPT ensemble for $100 \mathrm{ps}$, in which the temperature and pressure were controlled at $300 \mathrm{~K}$ and 0 bar, respectively. Then, we simulated deformation-controlled uniaxial tensile and compressive loading in an NPT ensemble, and simulated shearing deformation in an NVT ensemble. The target temperature was $300 \mathrm{~K}$ and the strain rate was $0.001 \mathrm{ps}^{-1}$. The pressure components in the loading direction and engineering strain were used to draw the stress-strain curve. The tensile strength of the model was determined by the maximum stress on the stressstrain curve, and the corresponding strain was determined as the fracture strain. During the deformation, the number of bonds was counted using a cutoff of $1.85 \AA$, which is a measure of bond cracking in the network. We also computed the coordination and carbon rings using homemade codes, and render the images in OVITO software. ${ }^{14}$ The atomic strain and atomic rotation were computed by the build-in modules of OVITO. ${ }^{14}$

Computation of Young's modulus. To determine the Young's modulus, we needed to calculate the elastic constants. The simulations were performed in an NVT ensemble and the temperature was fixed at $300 \mathrm{~K}$. We used the $1 \%$ strain to obtain the corresponding stress tensor. The average changes in stress tensor and applied strain were used to compute one row of elastic stiffness tensor. There were six deformation directions and six corresponding tensor components, resulting in 21 independent elastic constants in the elastic tensor. At last, these elastic constants were taken into the VoigtReuss-Hill equations to compute the Young's modulus. ${ }^{15}$ This computational method is mature in the elastic mechanics of solids. ${ }^{16} \mathrm{We}$ also noted that Tomas et al. calculated the Young's modulus of carbide-derived carbons. ${ }^{3}$ In our current work, the influence of annealing temperature on Young's modulus of final DGN structures was not considered. But the positive correlation between average angular defect and annealing temperature demonstrates that DGNs obtained from low annealing temperatures should exhibit higher Young's modulus and strength. In addition, the Zener ratio (Fig. S6) was calculated from the elastic constants to analyze the slight anisotropy of DGNs. 
Calculation of compressive modulus from compressive stress-strain curves. Since there are no apparent dividing points in compressive stress-strain curves, we calculated the average value of tangent modulus at 1\%,2\%,5\%, and $8 \%$ strains from each compressive stress-strain curve. Thus, in Fig. 2(c), the compressive modulus exhibits error bars.

Calculations of angular defects and XRD spectra. Angular defects (local curvatures) were calculated using the surface mesh-based method proposed by Martin et al. ${ }^{1}$ The average angular defect is the average value of all angular defects in the whole structure. ${ }^{1}$ We used the LAMMPS command (compute xrd) to calculate the XRD spectra of different DGNs. This command can be found on the website of LAMMPS homepage (https://docs.lammps.org/compute_xrd.html).

Strain rate sensitivity. In MD simulations, the strain rate sensitivity is a well-known problem due to the time scale and computational cost. In the previous study of graphene monolayer, ${ }^{17}$ it was found that the strain rate displays a negligible influence on the Young's modulus, fracture strain, and fracture strength of graphene at $300 \mathrm{~K}$. In our simulations, the strain rate $(\dot{\varepsilon})$ is $0.001 \mathrm{ps}^{-1}\left(=10^{9} \mathrm{~s}^{-1}\right) .{ }^{18}$ To test the influence of strain rate on mechanical properties of DGNs, we compared the tensile stress-strain curves of the DNG model $\left(\rho=1.12 \mathrm{~g} / \mathrm{cm}^{3}\right)$ with three different strain rates $\left(\dot{\varepsilon}=10^{7}, 10^{8}\right.$, and $10^{9}$ $\left.\mathrm{s}^{-1}\right)$. As shown in Fig. S7, the tensile stress-strain curves exhibit overlapped elastic stages and highly similar plastic stages. The values of Young's modulus for the three cases are the same, and the values of tensile strength for the three cases are very close $(\sigma=2.55 \pm 0.182 \mathrm{GPa})$. Thus, the strain rate used in our simulations is reasonable and the results of mechanical properties are reliable.

Repeatability of mechanical properties of DGNs in MD simulations. In Table S1, the smallest box length in our simulations is $110 \AA$, which should be sufficient to contain different topological microstructures. To test the repeatability, we obtained different initial structures with the same densities from annealing simulations. Considering the computational cost, we selected four densities $(\rho=0.33,0.56,1.12$, and $1.54 \mathrm{~g} / \mathrm{cm}^{3}$ ) as examples. As shown in Fig. S8, stress-strain curves for each density exhibit similar strength and failure strain. In some cases, the small difference of tensile modulus for each density should be attributed to the slight anisotropy (see the Zener ratio). For high-density DGNs, the anisotropy of peak tensile strength at three directions is mainly due to the anisotropy of multiple stacks of graphene ribbons. ${ }^{6}$ The calculation of Young's modulus has considered the slight anisotropy of DGN structures. Since the tensile stress-strain curve of each DGN has a long plastic platform with no sudden drop, the failure strains of DGNs defined in Fig. 5(b) are much conservative. We also added more simulated data points in Fig. 4(b) and Fig. 5(b). Young's modulus and strength (logarithms) still exhibit inversely-proportional relationships with the average angular defect. The location of DGNs in Fig. 5(b) is unaffected. Thus, in statistics, the phenomena reported in this work are repeatable. The statistical errors for strength and failure strain should have a negligible influence on our main conclusions. 


\section{References}

1. Martin J W, de Tomas C, Suarez-Martinez I, et al. Topology of disordered 3D graphene networks. Physical Review Letters, 2019, 123(11): 116105.

2. de Tomas C, Suarez-Martinez I, Vallejos-Burgos F, et al. Structural prediction of graphitization and porosity in carbide-derived carbons. Carbon, 2017, 119: 1-9.

3. de Tomas C, Suarez-Martinez I, Marks N A. Carbide-derived carbons for dense and tunable 3D graphene networks. Applied Physics Letters, 2018, 112(25): 251907.

4. de Tomas C, Suarez-Martinez I, Marks N A. Graphitization of amorphous carbons: A comparative study of interatomic potentials. Carbon, 2016, 109: 681-693.

5. Marks N A. Generalizing the environment-dependent interaction potential for carbon. Physical Review B, 2000, 63(3): 035401.

6. Wang Y C, Zhu Y B, Wu H A. Formation and topological structure of threedimensional disordered graphene networks. Physical Chemistry Chemical Physics, 2021, 23(17): 10290-10302.

7. Plimpton S. Fast parallel algorithms for short-range molecular dynamics. Journal of Computational Physics, 1995, 117(1): 1-19.

8. Stuart S J, Tutein A B, Harrison J A. A reactive potential for hydrocarbons with intermolecular interactions. The Journal of Chemical Physics, 2000, 112(14): 64726486.

9. Wei Y, Wu J, Yin $\mathrm{H}$, et al. The nature of strength enhancement and weakening by pentagon-heptagon defects in graphene. Nature Materials, 2012, 11(9): 759-763.

10. Liu F, Ming P, Li J. Ab initio calculation of ideal strength and phonon instability of graphene under tension. Physical Review B, 2007, 76(6): 064120.

11. Zhan H, Zhang Y, Yang C, et al. Graphene helicoid as novel nanospring. Carbon, 2017, 120: 258-264.

12. Xia K, Zhan H, Gu Y. Two-dimensional graphene heterojunctions: the tunable mechanical properties. Carbon, 2015, 95: 1061-1068.

13. Chen M W, Wu B, Zhou L C, et al. Micromechanical properties of pyrolytic carbon with interlayer crosslink. Carbon, 2020, 159: 549-560.

14. Stukowski A. Visualization and analysis of atomistic simulation data with OVITOthe Open Visualization Tool. Modelling and Simulation in Materials Science and Engineering, 2009, 18(1): 015012.

15. Hill R. The elastic behaviour of a crystalline aggregate. Proceedings of the Physical Society. Section A, 1952, 65(5): 349.

16. Timoshenko S P, Goodier J N. Theory of Elasticity (Third Edition). McGraw-Hill Press, 1970.

17. Zhao H, Aluru N R. Temperature and strain-rate dependent fracture strength of graphene. Journal of Applied Physics, 2010, 108: 064321.

18. Zhang X, Zhong L, Mateos A, et al. Theoretical strength and rubber-like behaviour in micro-sized pyrolytic carbon. Nature nanotechnology, 2019, 14(8): 762-769. 


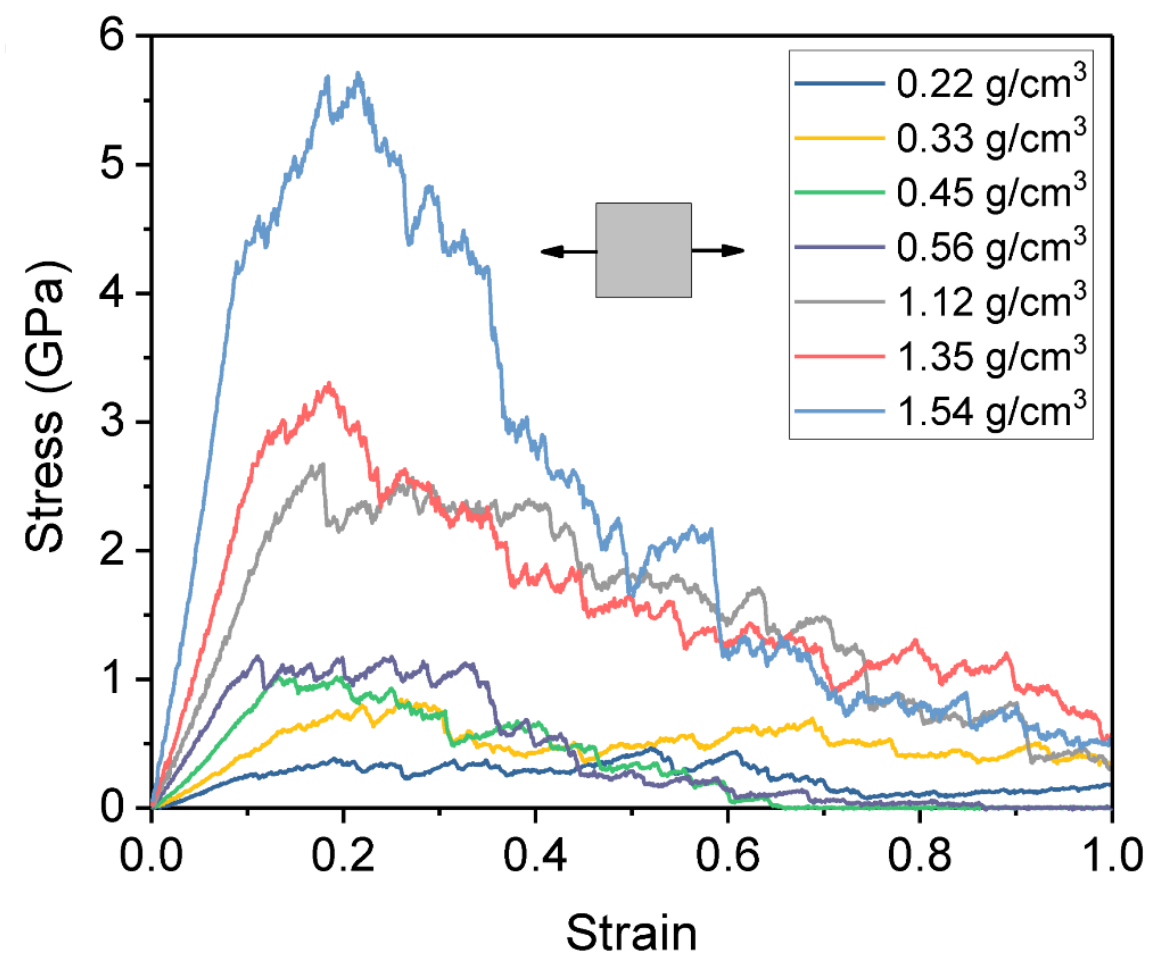

Figure S1. Tensile stress-strain curves of DGNs with different densities. 
(a) $\varepsilon=0$

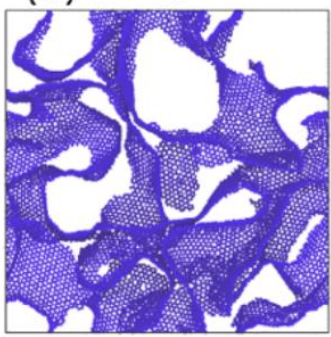

(d) $\varepsilon=0$

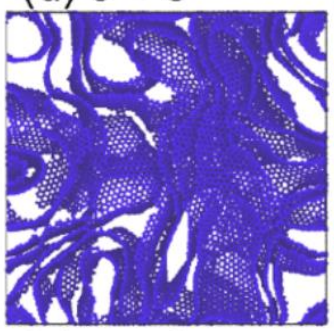

(g) $\varepsilon=0$

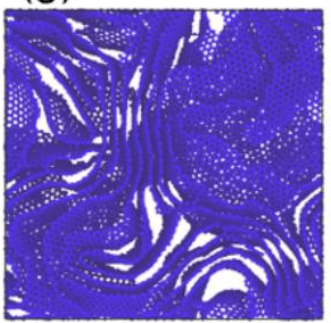

(j)

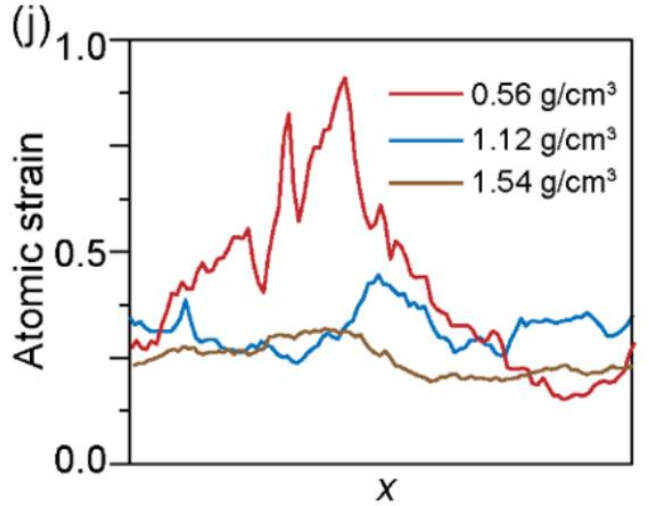

(h) $\varepsilon=0.15$ (c) $\varepsilon=0.36$
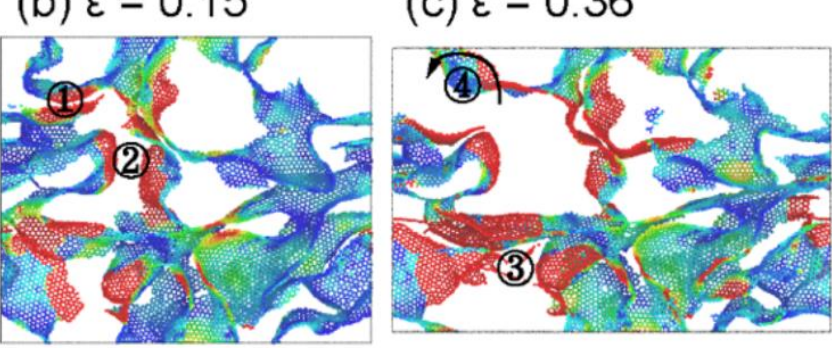

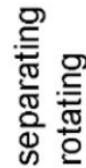

(ㄱ) $(9)$

(f) $\varepsilon=0.36$

을

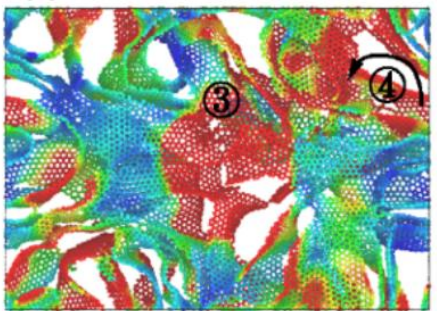

(i) $\varepsilon=0.36$
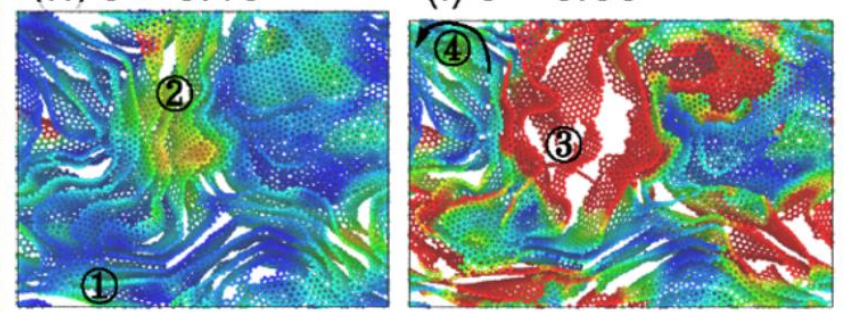

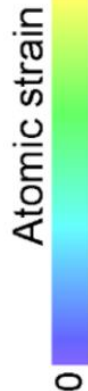

(k)

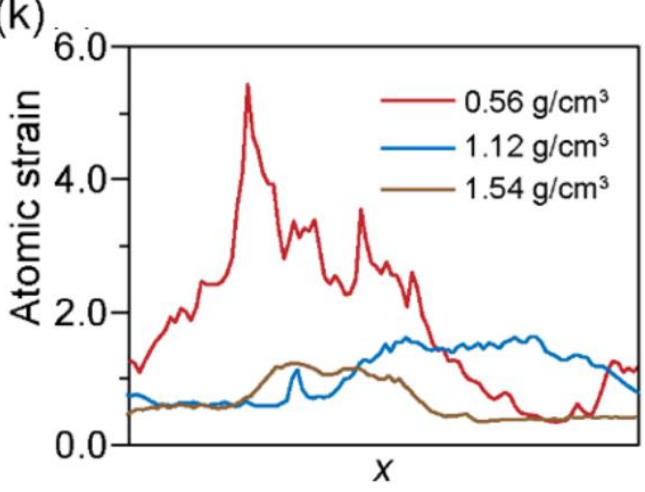

Figure S2. Atomic strain distributions and local deformation modes of stretched DGNs. (a-c) $\rho=0.56 \mathrm{~g} / \mathrm{cm}^{3}$. (d-f) $\rho=1.12 \mathrm{~g} / \mathrm{cm}^{3}$. (g-i) $\rho=1.54 \mathrm{~g} / \mathrm{cm}^{3}$. (j-k) Average atomic strain distributions of stretched DGNs $(\varepsilon=0.15$ and 0.36$)$ along the length direction. 
(a) $\varepsilon=0$

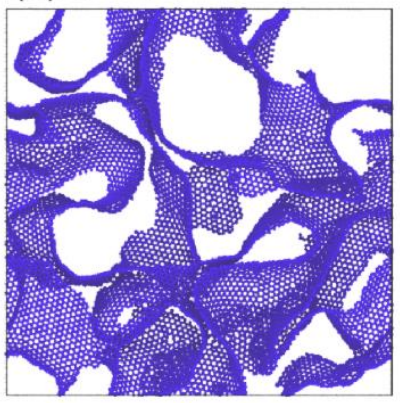

(d) $\varepsilon=0$

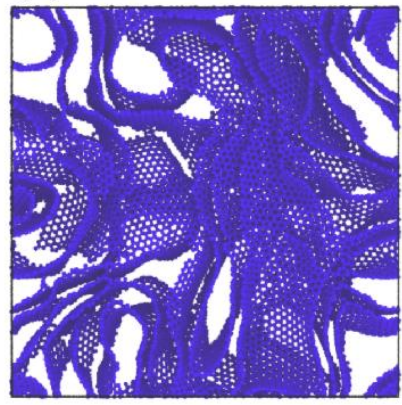

(b) $\varepsilon=0.2$

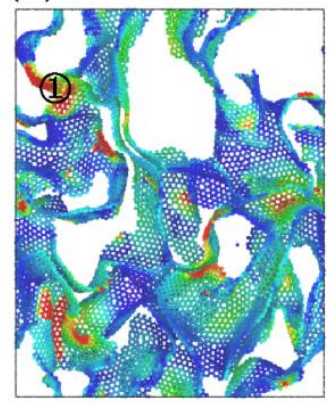

(e) $\varepsilon=0.2$

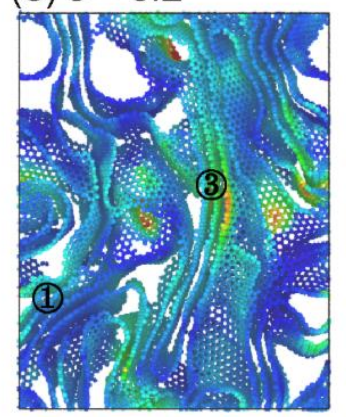

(c) $\varepsilon=0.4$

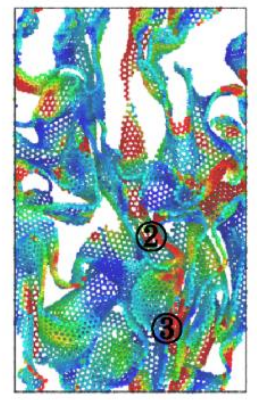

(f) $\varepsilon=0.4$

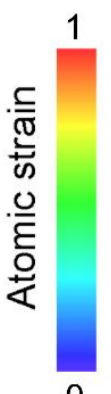

(1) sliding

(2) folding

(3) contacting

Figure S3. Atomic strain distributions and local deformation modes of compressed DGNs $\left(\rho=0.56\right.$ and $\left.1.12 \mathrm{~g} / \mathrm{cm}^{3}\right)$. 


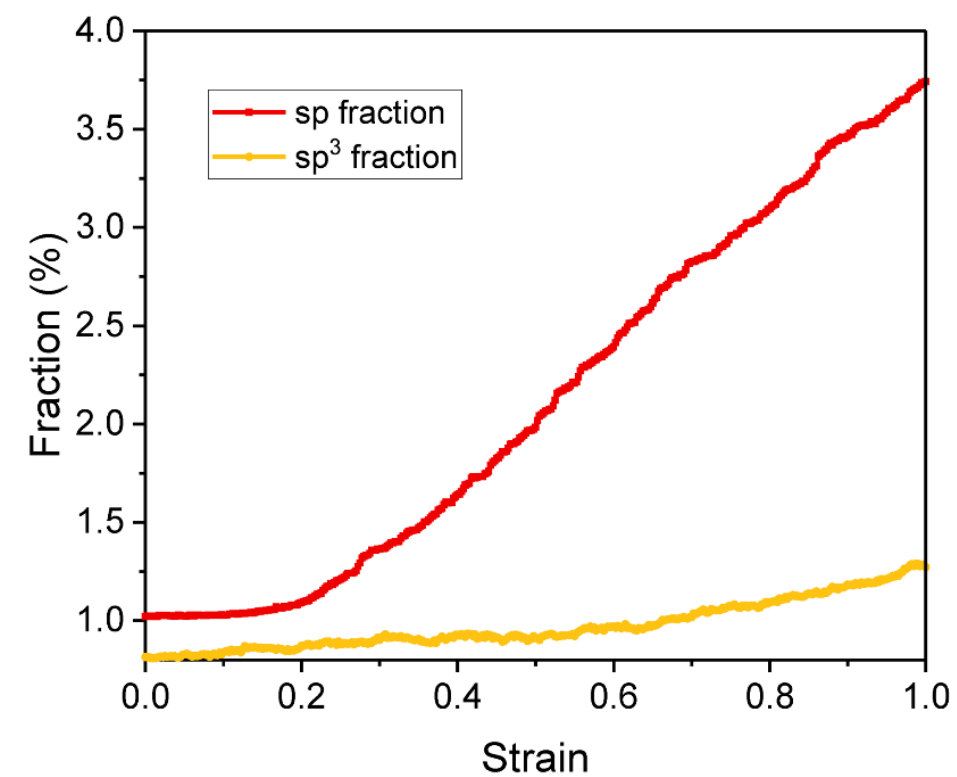

Figure S4. Fractions of $s p$ - and $s p^{3}$-bonded atoms as functions of shear strain. The density of the DGN is $1.12 \mathrm{~g} / \mathrm{cm}^{3}$. The increase of $s p$ fraction denotes the arising of torn graphene and cracked out-of-plane topological defects. 


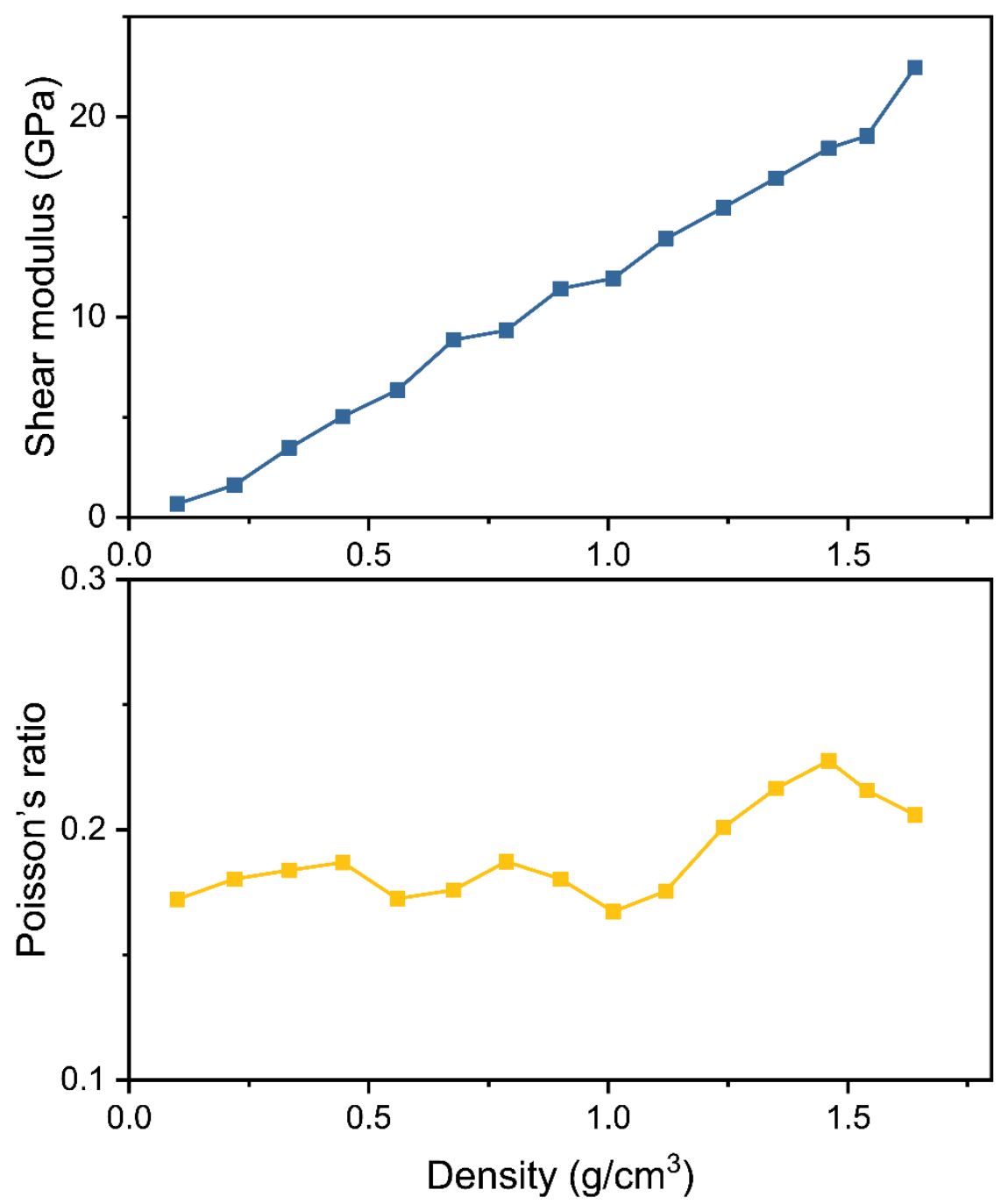

Figure S5. Shear modulus and Poisson's ratio as functions of the density. 

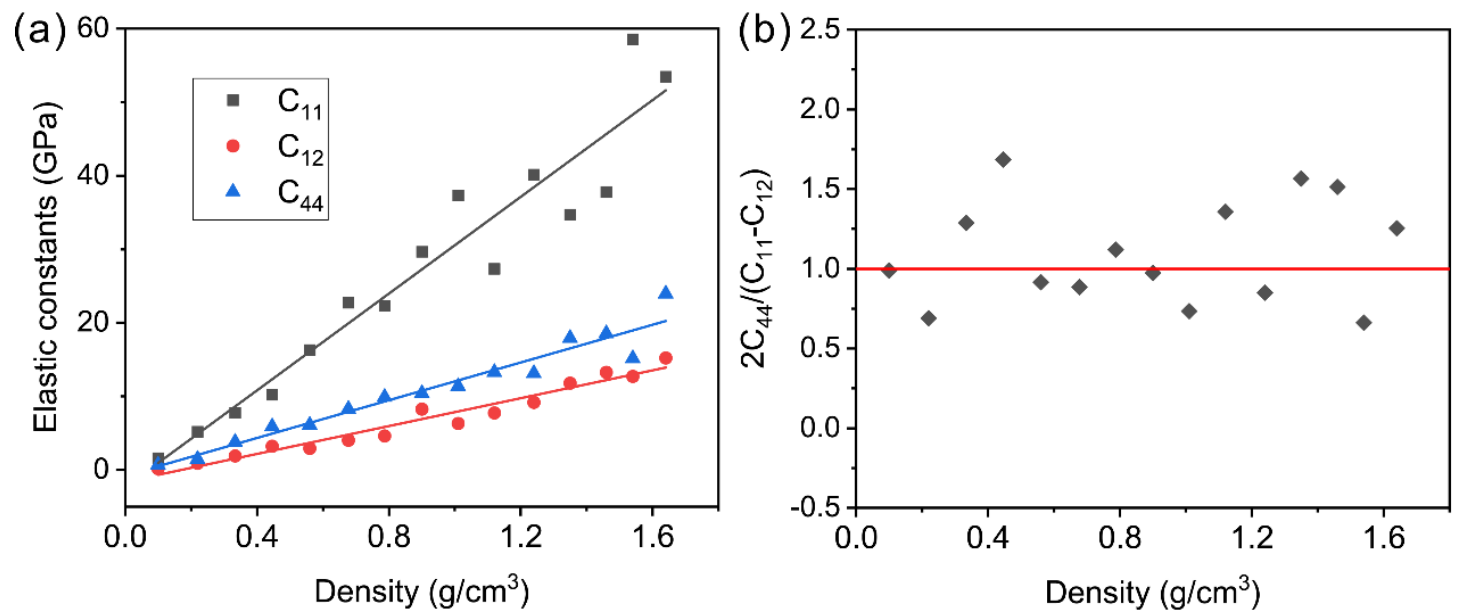

Figure S6. Analysis on the slight anisotropy of DGNs. (a) Elastic constants $\left(\mathrm{C}_{11}, \mathrm{C}_{12}\right.$, $\mathrm{C}_{44}$ ) as functions of the density. (b) Zener ratio of DGNs with different densities. For isotropic materials, the Zener ratio is demonstrated by the red line. ${ }^{14}$ In our simulations, the size of DGN models is sufficient to contain different topological microstructures. The calculated Zener ratio of DGNs is closed to the red line. Thus, DGNs only show slight anisotropy. 


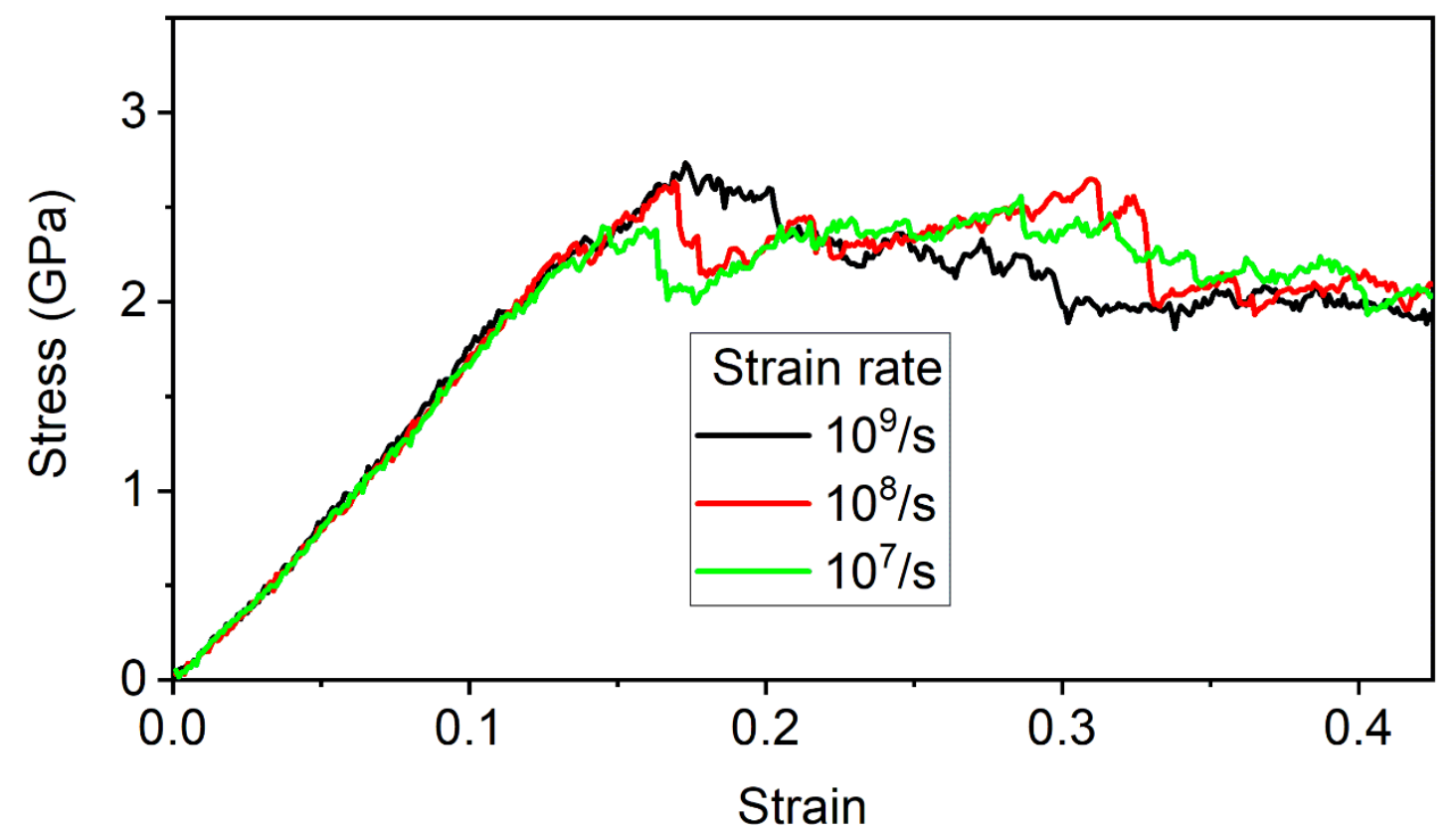

Figure S7. Tensile stress-strain curves of the DNG model $\left(\rho=1.12 \mathrm{~g} / \mathrm{cm}^{3}\right)$ with three different strain rates. 

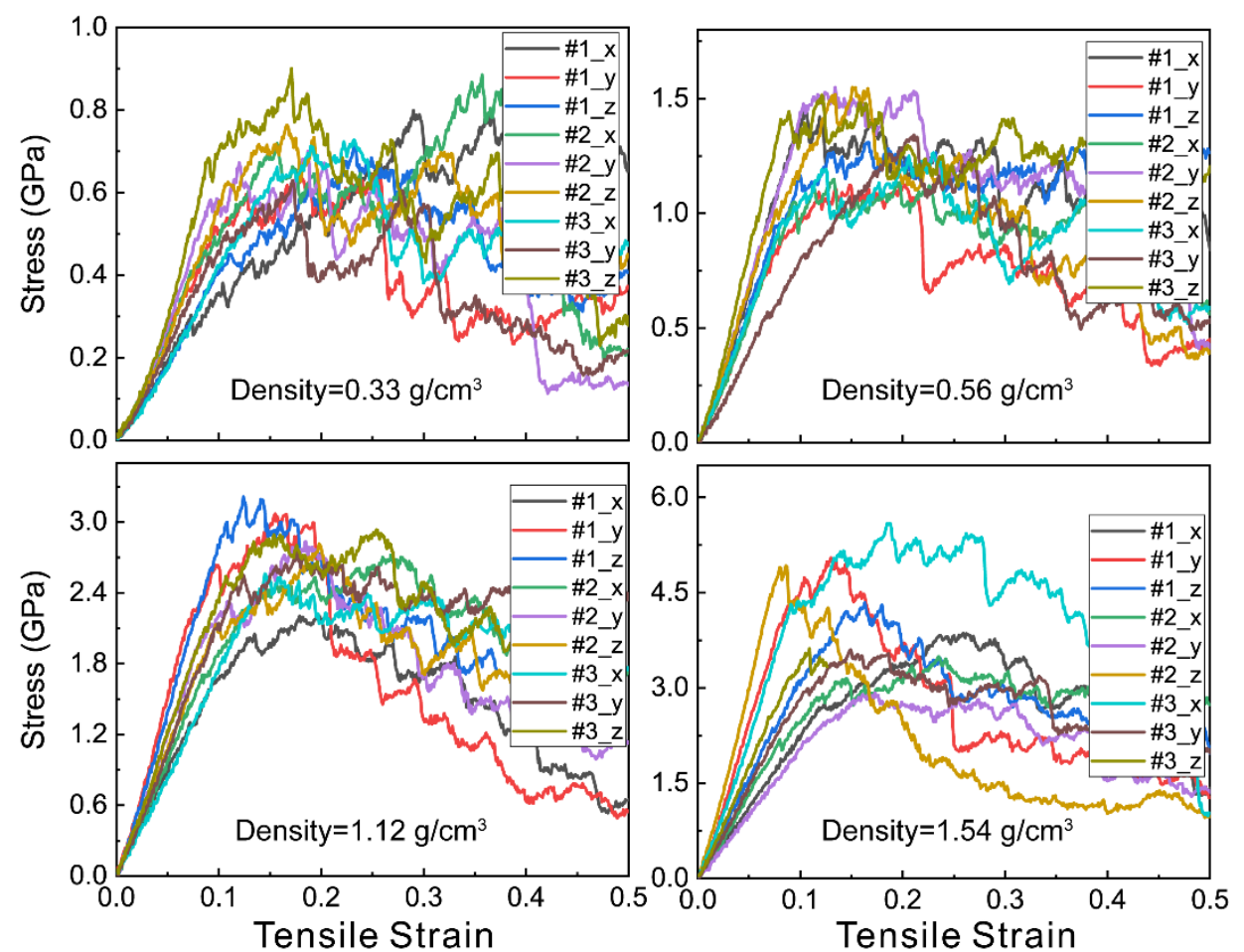

Figure S8. Tensile stress-strain curves of DGNs with different initial microstructures. Herein, four different densities $\left(\rho=0.33,0.56,1.12\right.$, and $\left.1.54 \mathrm{~g} / \mathrm{cm}^{3}\right)$ are considered as examples to discuss the statistical errors of mechanical properties. The calculation of Young's modulus in Fig. 4(a) has considered the slight anisotropy of DGN structures. With the increase of density, more multiple stacks of graphene ribbons can be found in DGNs, which would induce the anisotropy of peak tensile strength at three directions. Failure strains of DGNs defined in Fig. 5(b) are much conservative due to the long plastic-like stage in tensile stress-strain curves. More simulated data points are added in Fig. 4(b) and Fig. 5(b), which do not influence the novel scaling laws between mechanical properties and structural topology and the locations of DGNs in Ashby chart of specific strength versus fracture strain. 